\title{
Dietary omega-3 fatty acids aid in the modulation of inflammation and metabolic health
}

\author{
by Angela M. Zivkovic, Natalie Telis, J. Bruce \\ German and Bruce D. Hammock
}

This article focuses on the role of omega-3 fatty acids as precursors for lipid signaling molecules known as oxylipins. Although omega-3 fatty acids are beneficial in autoimmune disorders, inflammatory diseases and heart disease, they are generally underrepresented in the American diet. A literature review confirms that the consumption of omega-3 fatty acids - whether in food sources such as walnuts, flax seeds and fatty fish (including salmon and sardines), or in supplements - is associated with decreased morbidity and mortality. This growing body of evidence, including the results of a recent study of patients with kidney disease, highlights the need to measure omega-3 fatty acids and their oxylipin products as markers of metabolic health and biomarkers of disease. In addition, there is substantial evidence of the need to increase the omega-3 fatty acid content of American diets to optimize metabolic health.

M any of the most significant U.S. health concerns today are modulated by omega-3 fatty acids, particularly eicosapentaenoic acid (EPA) and docosahexaenoic acid (DHA). Omega-3 fatty acids are associated with the prevention or reduction in severity of a multitude of diseases, from metabolic diseases such as heart disease, diabetes and kidney disease to neurodegenerative diseases such as Alzheimer's to an array of other inflammatory diseases including osteoarthritis.

EPA and DHA attenuate the development of atherosclerosis, or arterial plaques, by reducing concentrations of inflammatory signaling molecules called cytokines and adhesion molecules at the arterial wall where plaque forms (De Caterina et al. 2004). EPA and DHA

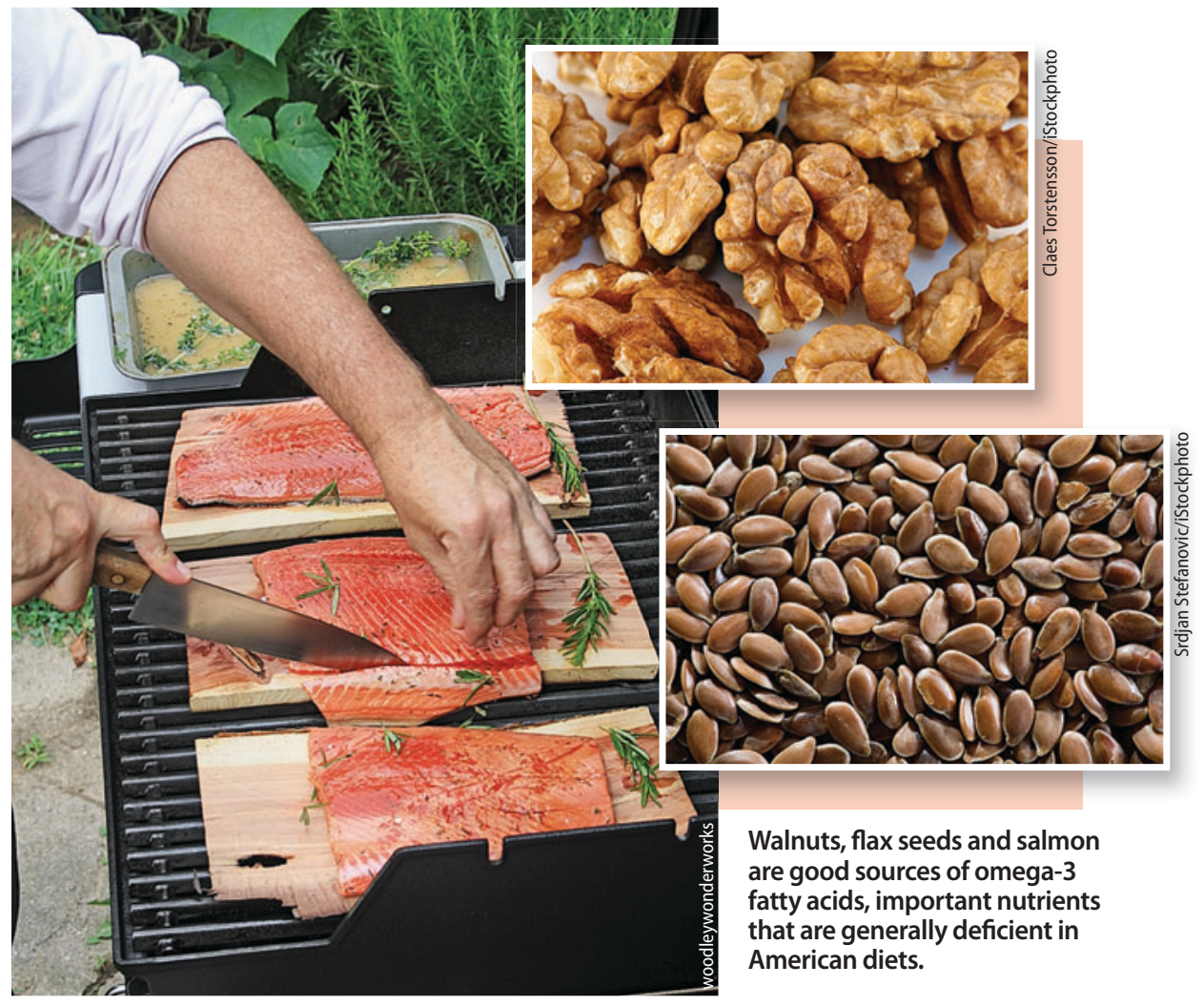

have also been shown to stabilize atherosclerotic plaques, thereby reducing the likelihood of fatal and nonfatal cardiovascular events (Thies et al. 2003). EPA and DHA additionally reduce the synthesis of triglycerides (fat molecules) and secretion from the liver, and increase the size of low-density lipoproteins, which contribute to the reduction of cardiovascular disease risk (Griffin et al. 2006). EPA and DHA improve liver health by reducing steatosis (accumulation of fat in the liver) in patients with nonalcoholic fatty liver disease (Capanni et al. 2006). They also improve kidney health by attenuating or even reversing the loss of kidney function and reducing hypertension in kidney diseases involving the glomerulus, the main filtering part of the kidney (Donadio et al. 1994). Omega-3 fatty acids affect the joints and are used as analgesics or pain reducers in rheumatoid arthritis (Goldberg and Katz 2007).

The omega-3s even play a role in brain health: high blood plasma levels of omega-3 fatty acids are associated with a reduced risk of neurodegenerative diseases such as Alzheimer's disease (Schaefer et al. 2006) and mental disorders such as schizophrenia (McNamara et al. 2007) and depression (Sanchez-Villegas et al. 2007). Taken in supplement or food form, omega- 3 fatty acids have been found to reverse the progression of a number of inflammatory diseases, from inflammatory bowel disease to diseases of the skin and joints, to other autoimmune diseases such as lupus and multiple sclerosis (Simopoulos 2002). This review focuses on the basic biology of omega-3 fatty acids as nutritional modulators of inflammation and presents preliminary results of a study of oxylipin biomarkers in kidney disease patients.

\section{Intake, food sources and metabolism}

Saturated and monounsaturated fatty acids, which have no double bonds or a single double bond, respectively, can be synthesized in the liver. In contrast,

Online: http://californiaagriculture.ucanr.org/ landingpage.cfm?article=ca.v065n03p106\&fulltext=yes DOl: 10.3733/ca.v065n03p106 
polyunsaturated omega- 3 and omega- 6 fatty acids, which have multiple double bonds, are considered essential in the human diet: they cannot be synthesized and must be consumed. Terrestrial plants contain the omega- 6 fatty acid linoleic acid and the omega-3 fatty acid alpha-linolenic acid but not the long-chain metabolites arachidonic acid, or EPA and DHA. Both linoleic acid and alpha-linolenic acid can be converted to their respective longchain metabolites in the liver through a series of enzymatic conversions (fig. 1).

However, since omega- 3 and omega- 6 fatty acids compete for the same enzymes, the relative dietary proportions of precursor fatty acids determine the net rate of conversion to their respective long-chain derivatives (Goyens et al. 2006; Liou et al. 2007). Linoleic acid is readily converted to its long-chain metabolite arachidonic acid, and this conversion is driven by the amount of linoleic acid ingested. However, most humans convert a smaller proportion of ingested alphalinolenic acid into EPA and even less into DHA (Pawlosky et al. 2003). Most studies agree on estimates of about $5 \%$ conversion to EPA and less than 1\% conversion to DHA (Burdge and Calder 2006). Due to this low rate of conversion of alphalinolenic acid to EPA and DHA, and a generally low consumption of preformed dietary long-chain omega-3 fatty acids, many Americans are likely deficient in EPA or DHA.

Omega- 6 fatty acids are abundant in the Western diet and are found in high proportions in most cooking oils, grains and grain-fed animal products. On the other hand, omega-3 fatty acids are generally deficient in the foods typically

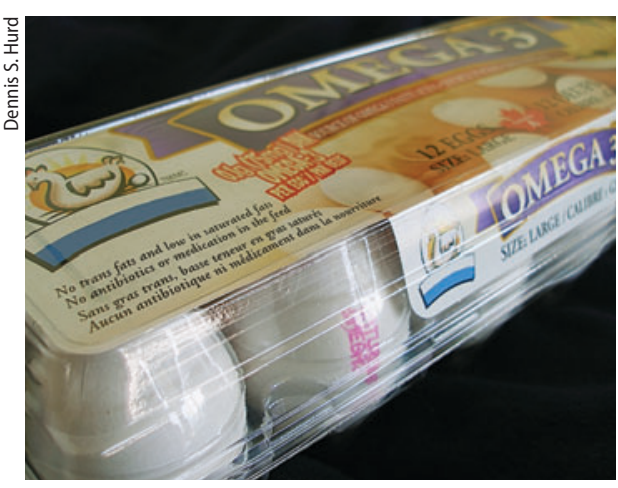

Eggs from chickens that have been fed flax have higher concentrations of omega-3 fatty acids, which have been shown in a number of studies to be beneficial to human health. consumed by Americans. Certain foods - including leafy greens, walnuts, canola oil, flax-fed chicken eggs and fatty fish (e.g., wild salmon, anchovies, mackerel and tuna) - have relatively large concentrations of omega-3. Historically, humans consumed diets with much higher relative proportions of omega-3, with ratios of omega- 6 to omega- 3 of 1-to-1 or 2-to-1, in contrast to modern diets with ratios as high as 15 -to-1 to 25 -to-1 (Simopoulos 2008).

The disproportionate consumption of omega- 6 as compared to omega- 3 fatty acids is the result of common U.S. dietary patterns. Foods such as salmon, walnuts, kale and eggs from chickens fed flax have higher omega-3 fatty acid content than do more common American foods, like corn oil, beef and potatoes (USDA 2011). For example, raw pink salmon has 419 milligrams of EPA and 586 milligrams of DHA per 100 grams, whereas raw ground beef contains none. Walnuts contain 3,800 milligrams of linoleic acid and 9,080 milligrams of alpha-linolenic acid per 100 grams. On the other hand, peanuts contain 15,600 milligrams of linoleic and only 3 milligrams of alpha-linolenic acid per 100 grams. These ratios mean that the typical American diet is deficient in omega-3 fatty acids, especially when considered in relation to omega- 6 fatty acids (Kris-Etherton et al. 2000).

Because of the increasing levels of contaminants, like mercury and dioxins, in both domestic and imported fish (Stahl et al. 2009; Sunderland 2007), the consumption of fish as a main source of long-chain omega-3 fatty acids is problematic, and some types of fish are not recommended for consumption by pregnant women and small children (Jedrychowski et al. 2007). Since terrestrial plants contain only the short-chain omega-3 precursor alpha-linolenic acid, and since alphalinolenic acid is poorly converted into its long-chain metabolites EPA and DHA, it is likely that the American diet will continue to be relatively deficient in these important molecules unless significant dietary changes are made.

\section{Reducing disease risks}

Cardiovascular disease. Omega-3 fatty acids play an important role in reducing cardiovascular disease, a leading cause of death in the United States (Harris et al. 2009). They reduce events that lead

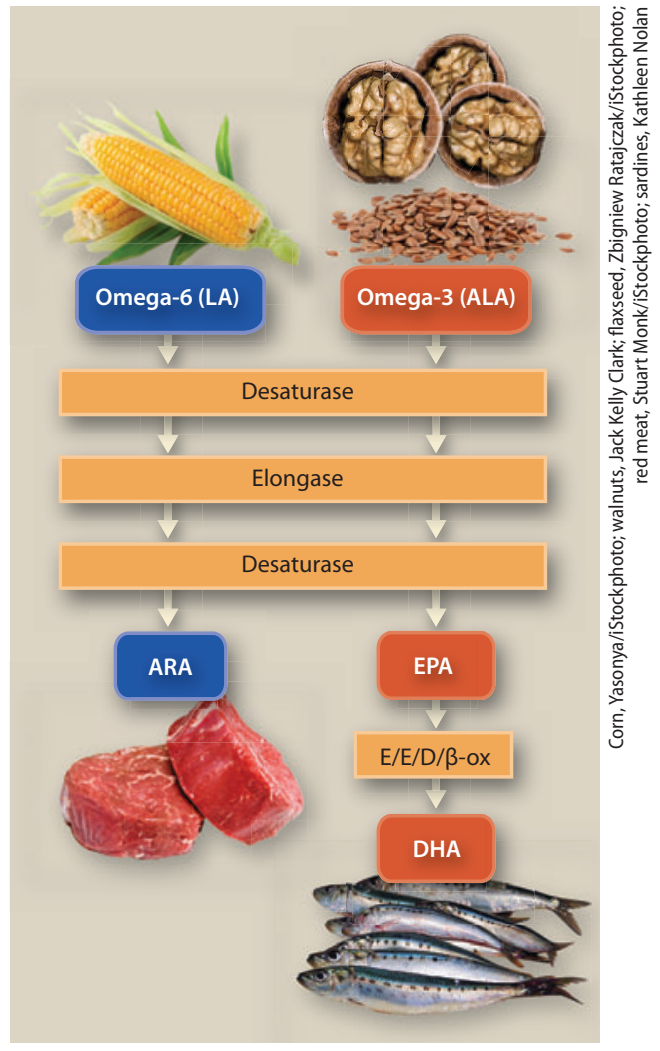

Fig. 1. Dietary sources and enzymatic conversion pathways of omega-3 and omega- 6 fatty acid precursors. The omega- 3 precursor alphalinolenic acid (ALA) is found in walnuts and flax seeds, and the omega- 6 precursor linoleic acid (LA) is found in corn and vegetable oils. Both are converted by desaturase and elongase enzymes into their long-chain derivatives: ALA is converted to eicosapentaenoic acid (EPA) and docosahexaenoic acid (DHA); and LA is converted to arachidonic acid (ARA). ARA is found in animal products, such as beef, and EPA and DHA are found in fatty fish, such as sardines.

to heart attack by stabilizing the heart muscles against arrhythmia (irregular heartbeat or abnormal heart rhythm) (Ballantyne 1999). For example, 200 milligrams DHA per day decreases the chance of death from cardiac arrest by $50 \%$ (Horrocks and Yeo 1999).

EPA and DHA metabolites stimulate the dilation of arterioles (small arteries) (Ye et al. 2002), which decreases blood pressure and inhibit the expression of inflammatory genes (Bouwens et al. 2009). A literature review covering 2002 to 2004 - including a pooled analysis of 48 randomized, controlled trials and $41 \mathrm{co}-$ hort trials - found no significant benefit of supplementation with omega-3 fatty acids for the reduction of total mortality, cardiovascular events or cancer (Hooper et al. 2004). However, as the authors point out, there was "significant statistical 
heterogeneity" among the results given that the dosages and formulations of omega-3 fatty acids and the outcomes evaluated varied substantially, with some studies showing benefits and others not. This suggests that further studies are needed to evaluate the specific effects of different dosages and formulations of omega-3 fatty acids in different population groups and at different supplementation levels.

Kidney disease. Animal trials show that omega-3 fatty acid treatment decreases blood pressure, has anti-inflammatory effects, slows renal failure and moderates the side effects of hypertension (Imig et al. 2005; Zhao et al. 2004). This has been the basis for the study of the effects of omega- 3 fatty acids on kidney disease. A recent literature review found that no definitive conclusions can be made about the effectiveness of omega- 3 fatty acids for the prevention or treatment of kidney disease (Fassett et al. 2010).

However, just as in the review of effects on cardiovascular disease, the authors point out that there was substantial variability from study to study in the dosages, proportions of specific omega-3 fatty acids administered (i.e., formulations), duration of supplementation, sample sizes and specific outcomes assessed. This again suggests that further studies are needed to better understand the specific formulations, dosages and markers of effectiveness for omega-3 fatty acid supplementation.

Neurological disease. There is increasing evidence that DHA specifically plays a significant role in neurological development and disease prevention. DHA is an important component of brain development, and DHA deficiencies have been found in patients with neurological conditions. For example, there is a strong correlation between depression and DHA deficiency (Horrocks and Yeo 1999). Deficiency in DHA has also been strongly correlated with neurodegenerative diseases common in the elderly population, particularly Alzheimer's disease (Freemantle et al. 2006).

Arthritis. Omega-3 fatty acid intake is associated with the improvement of rheumatoid arthritis, as omega- 3 metabolites inhibit the production of inflammatory cytokines responsible for arthritic pain (Zainal et al. 2009). Supplementation with
EPA and DHA is effective against arthritic pain as well as other symptoms, including joint stiffness (Goldberg and Katz 2007).

\section{Oxylipins and inflammation}

Oxylipins are fatty acids that have been converted into lipid signaling molecules by enzymes in the body. The omega- 6 fatty acid arachidonic acid, and the omega-3 fatty acids EPA and DHA, are converted into a wide variety of oxylipins with diverse and important signaling pathways that mediate a number of biological functions (Funk 2001).

Most of the functions of oxylipins derived from arachidonic acid, also called eicosanoids, are well known and primarily associated with immuno-stimulatory events (which stimulate the immune system) and pro-coagulant actions (which promote blood coagulation). However, the actions of these oxylipins are typically oversimplified as "pro-inflammatory," when, in fact, over 90 bioactive omega-3 and omega- 6 oxylipins with both pro- and anti-inflammatory effects are produced by enzymes - cyclooxygenase (COX), lipoxygenase (LOX) and cytochrome P450 (CYP).
Pro-inflammatory, pro-coagulant and immuno-stimulatory oxylipins are produced acutely in response to a diverse array of stimuli, such as injury to a blood vessel wall in order to initiate repair and immune cell recruitment, or to initiate blood clotting in response to injury to prevent hemorrhage (fig. 2). An injury to the vessel wall leads to an influx of calcium ions into the cell, which in turn stimulates the action of an intracellular enzyme that cleaves fatty acids from phospholipids (complex lipids that make up the cell membrane). This is followed by conversion of the released fatty acid into oxylipins, which initiate the recruitment of immune cells and stimulate signaling pathways to repair the injury via paracrine (signaling between neighboring cells) and autocrine (signaling within the same cell) pathways. Finally, after the injury has been repaired, the released fatty acids are converted into oxylipins that terminate the immuno-stimulatory and repair mechanisms.

The interplay of the various oxylipins produces results in nuanced responses to specific events over the course of an injury such that the net physiological effect

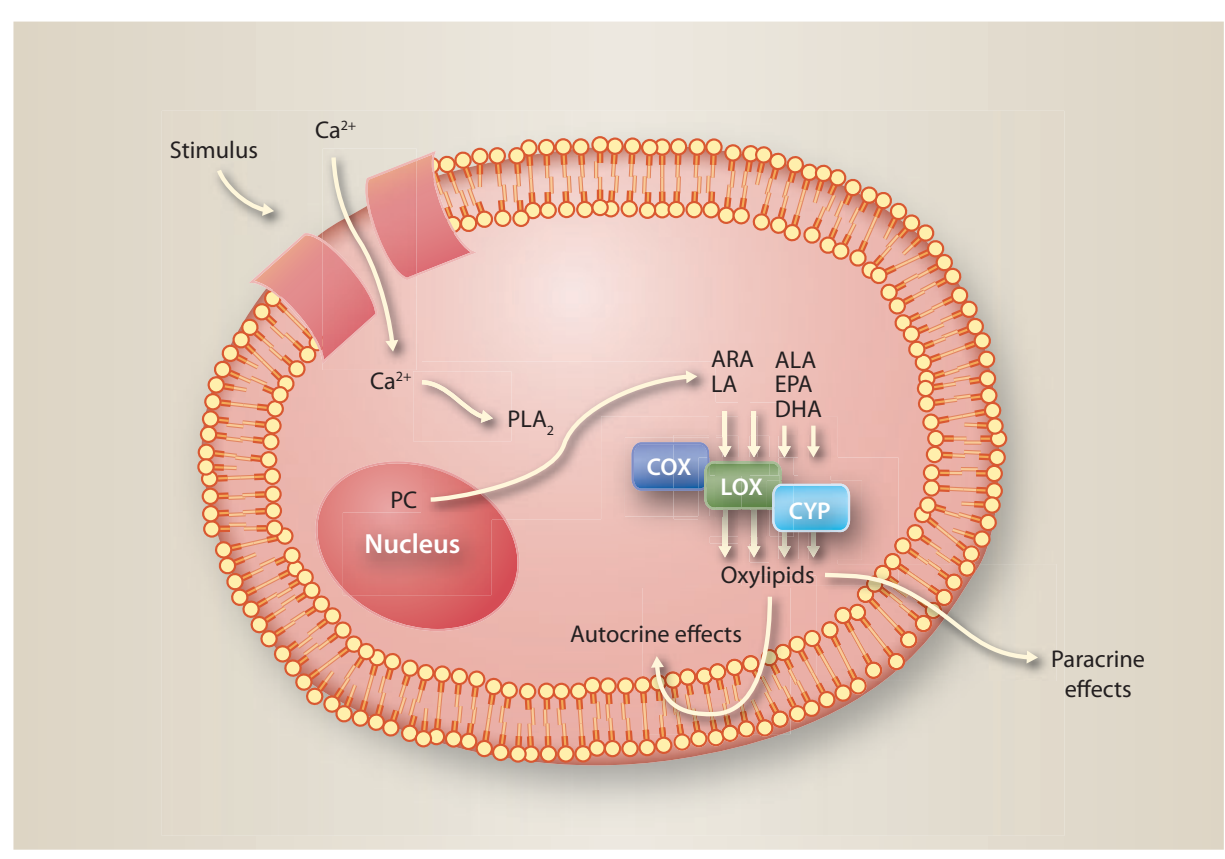

Fig. 2. Cellular mechanisms involved in producing oxylipin derivatives of omega- 3 and omega- 6 fatty acids. A stimulus such as cell wall injury activates transporter proteins, moving $\mathrm{Ca}^{2+}$ ions into the cell. This stimulates the release of fatty acids from nuclear membrane phospholipids (phosphatidycholine [PC]) by the enzyme phospholipase A2 (PLA $)$, and the conversion of omega-3 (eicosapentaenoic [EPA], docosahexaenoic [DHA] and alpha-linolenic [ALA] acid) and omega- 6 (arachidonic [ARA] and linoleic [LA] acid) fatty acids into signaling oxylipin products via the COX (cyclooxygenase), LOX (lipoxygenase) and CYP 450 (cytochrome P450) enzymes. These oxylipins are signaling molecules that help regulate inflammation, increasing and decreasing this important immune response within the cell (autocrine) and in neighboring cells (paracrine). 
of the complex combination of oxylipins is key, rather than the absolute concentration of any one of the oxylipins alone (Serhan and Savill 2005). Because oxylipins initiate and terminate various immune responses in different body tissues, they are important players in diseases such as heart disease, chronic inflammation, autoimmune disorders and cancer.

The dietary imbalance between omega- 6 and omega- 3 fatty acids can result in inappropriately elevated or sustained immuno-stimulatory responses to injury or inappropriately diminished immuno-suppressing responses after an injury has been repaired. It has been suggested that the increasing incidence of hypertensive and inflammatory conditions corresponds to the proportional increase in omega- 6 fatty acids and decrease in omega-3 fatty acids in the American diet (Simopoulos 2002).

\section{Assessment of omega-3 status}

Despite growing evidence supporting the beneficial effects of omega-3 fatty acids, there is little basic science about its metabolism under different lifestyle conditions and its variation across different populations and genotypes (genetic backgrounds). An adequate daily intake for adults of alpha-linolenic acid has been established by the U.S. Department of Agriculture at 2.22 grams per day based on consensus among field experts, and recommended EPA and DHA intake totals are 0.65 gram per day (Simopoulos et al. 1999). However, the biological endpoints for which omega-3 fatty acids are effective have not been established. For example, although omega-3s are known to prevent cardiac arrhythmia and heart attacks (Horrocks and Yeo 1999), a plasma level of omega-3 considered adequate for this effect has not been determined. Clearly, the measurement of omega-3 status would be beneficial in determining which individuals may be at increased risk of heart attack, as well as a number of other disorders that they mediate.

Even less is known about the optimal plasma concentrations of oxylipins. The normal ranges of omega-3-derived oxylipins were recently reported before versus after supplementation in a small sample of healthy men (Shearer et al. 2009). This is the first such investigation, and while little is known about the concentrations of omega-3 fatty acids

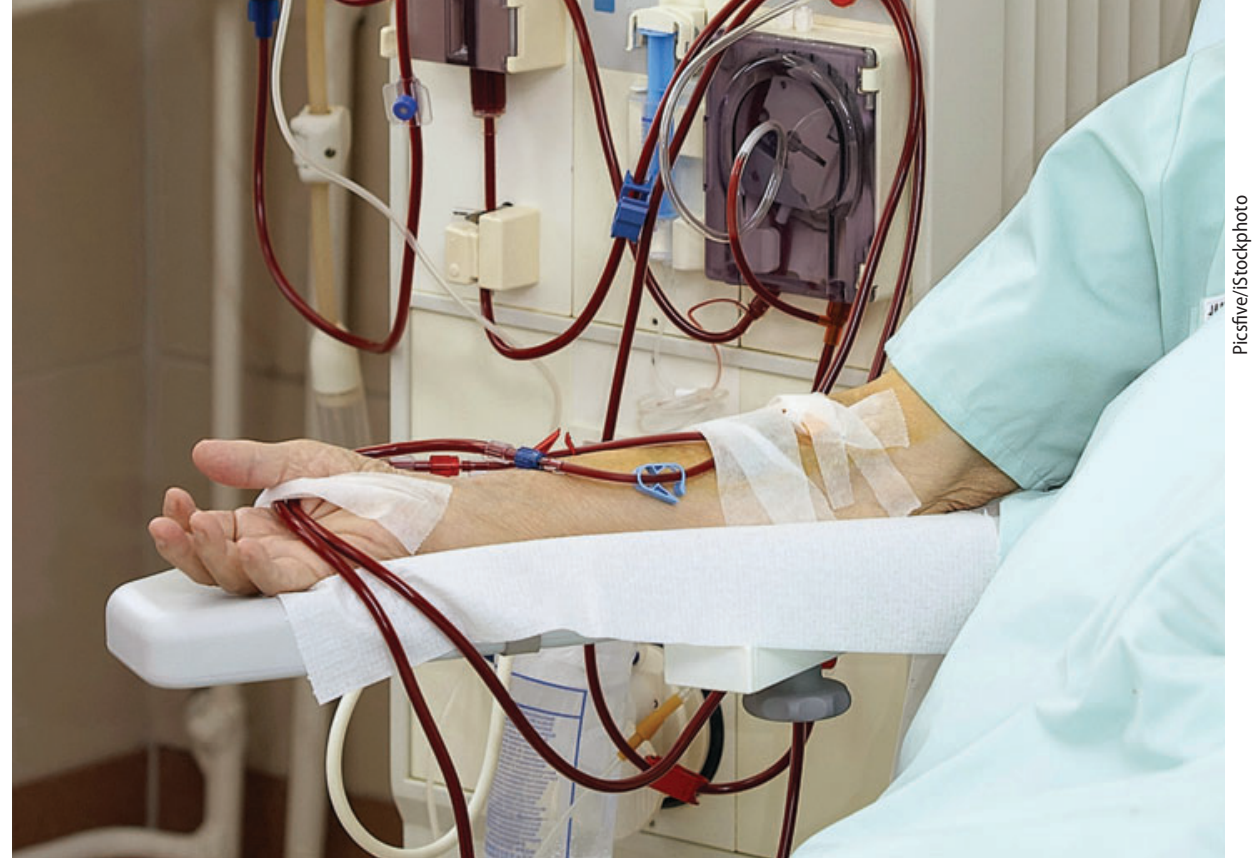

Evidence is mounting that omega- 3 fatty acids can moderate inflammation in the body and help reduce the risk and symptoms of kidney disease, heart disease and arthritis. Above, a patient undergoes dialysis for kidney disease.

themselves across populations, even less is known about concentrations of their oxylipin metabolites. The accurate measurement in plasma and red blood cells of omega- 3 and omega- 6 fatty acids, and the oxylipins derived from them, would enable the investigation of how these concentrations are affected by different disease states. Much like the measurement of cholesterol concentrations in plasma has led to reductions in the incidence of heart disease (EAS 1987), and the measurement of arachidonic acid-derived oxylipins has enabled the estimation of inflammation (Funk 2001), the measurement of omega-3 fatty acids and their oxylipin products will be valuable as a clinical toolset for detecting, monitoring and reversing an array of diseases. However, oxylipin measurement is currently limited to the research laboratory and is not available to the medical community or the general public as a diagnostic.

\section{Kidney disease study}

One example of the type of information obtainable by measuring oxylipin levels in humans was illustrated by a recent study of patients with immunoglobulin A nephropathy (IgAN), a type of inflammatory kidney disease. Because inflammation is one of the underlying causal mechanisms in IgAN, and because omega-3 fatty acids and their oxylipin products tend to be anti-inflammatory, a study was conducted to evaluate the effectiveness of omega-3 fatty acids in modulating kidney function in a set of IgAN patients.
In the current study, we identified a unique opportunity to measure oxylipins in a set of stored samples from a previously conducted trial of IgAN patients and to compare those to oxylipins in healthy control subjects. This was unique because the former patient trial involved 37 nephrology centers and was the culmination of over 8 years of work and millions of dollars in funding, which would have been nearly impossible to replicate.

For the stored samples from IgAN patients, the study design has been described in detail (Hogg et al. 2006). Briefly, 96 patients were recruited and randomly assigned to receive one of three daily treatments over 2 years: (1) a steroidal anti-inflammatory drug, (2) fish oil containing 2 grams EPA plus 1.5 grams DHA or (3) a corn oil placebo. Subjects had biopsy-confirmed IgAN, and kidney function was assessed as changes in glomerular filtration rate and urine protein to creatinine $(\mathrm{UP} / \mathrm{C})$ ratios.

The healthy control subjects were supplemented for 6 weeks with 2 grams EPA plus 1.5 grams DHA daily to match the concentrations of active ingredients (EPA and DHA) given to the IgAN patients. Controls were supplemented for 6 weeks instead of 2 years because as healthy subjects their kidney function was not assessed and because preliminary trials in our labs showed that 6 weeks was needed to achieve maximal plasma levels of omega-3 fatty acids at this supplementation dose.

Over 80 different individual oxylipin molecules were analyzed by mass 
spectrometry and grouped into categories based on their synthetic pathways. Oxylipins that are metabolized by the CYP enzymes are called epoxides, which can be further metabolized by the soluble epoxide hydrolase enzyme into diols. Oxylipins that are metabolized by the COX enzymes and those metabolized by

\section{Oxylipins may be a potential marker of improvement in kidney function and response to treatment.}

the LOX (5-LOX and 12/15-LOX) enzymes were added together. Total oxylipins were also calculated by summing all 87 individual oxylipins together.

Oxylipin responses. The IgAN patients had significantly higher total oxylipins as well as each of the categories of oxylipins - including epoxides, diols, COX and LOX metabolites - relative to healthy controls (table 1). These results are consistent with the systemic inflammation in $\operatorname{IgAN}$ patients that is associated with overproduction of oxylipins. These oxylipins are likely mediators of a vast array of inflammatory signaling events.

When the response to supplementation with omega-3 fatty acids was compared among IgAN patients and controls, there were clear differences (table 2). Whereas diols increased in controls by $27 \%$, they decreased by $17 \%$ in IgAN patients supplemented with fish oil. In controls, the COX metabolites decreased by $4 \%$ in

\begin{tabular}{|c|c|c|c|}
\hline \multirow[b]{2}{*}{ Oxylipin } & \multirow[b]{2}{*}{ Controlst } & \multicolumn{2}{|c|}{ IgAN patients } \\
\hline & & $\begin{array}{l}\text { Corn oil } \\
\text { placebo }\end{array}$ & Fish oil \\
\hline \multicolumn{4}{|c|}{ 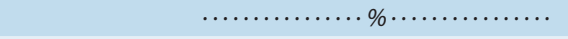 } \\
\hline Diols & 27 & 0 & -17 \\
\hline $\operatorname{cox} \neq$ & -4 & 0 & 0 \\
\hline Total oxylipins & 0 & 0 & -15 \\
\hline \multicolumn{4}{|c|}{$\begin{array}{l}\text { * Data are percent change from pre- to postsupplementation, } \\
\text { calculated as mean values of total oxylipins of each category for } \\
\text { each group ((Post-Pre)/Pre) } \times 100 \text {. }\end{array}$} \\
\hline \multicolumn{4}{|c|}{$\begin{array}{l}\text { † Controls = supplemented with omega-3 fatty acids from fish } \\
\text { oil; Fish oil = immunoglobulin A nephropathy (IgAN) patients } \\
\text { supplemented with omega-3 fatty acids from fish oil; Corn oil = } \\
\text { IgAN patients supplemented with corn oil placebo. }\end{array}$} \\
\hline \multicolumn{4}{|c|}{$\neq \mathrm{COX}=$ cyclooxygenase. } \\
\hline
\end{tabular}

response to fish oil, whereas in IgAN patients they did not change. Total oxylipins decreased by $15 \%$ in IgAN patients on fish oil but did not change in the controls.

These data indicate that IgAN patients responded differently to omega-3 fatty acid supplementation than did healthy controls. Since the IgAN patients had almost 200-fold higher total oxylipins than the controls at baseline, the $15 \%$ decrease in response to fish oil suggests that although fish oil was able to reduce inflammation in these patients, it was not enough to reduce their oxylipin levels to those of controls. As expected, oxylipin levels did not change in IgAN patients on the corn oil placebo.

Kidney function. The patients were also examined as two separate groups: those whose kidney function improved (greater than $25 \%$ decrease in UP/C) over 2 years versus those whose kidney function did not improve (less than a 25\% decrease in $\mathrm{UP} / \mathrm{C})$. In patients whose kidney function improved, total LOX metabolites were lower at baseline, and total COX metabolites were lower at the post time point (table 3). These data indicate that oxylipins may be a potential marker of improvement in kidney function and response to treatment.

\section{Targeted nutrition recommendations}

Once the normal ranges of omega-3 fatty acids and their oxylipin metabolites have been established, metabolite databases necessary for the discovery of specific plasma-based biomarkers will be used both to assess initial metabolic status and monitor treatment progress. Plasma oxylipin profiles could eventually be used to determine both inflammatory status and responsiveness to dietary changes in the ratio of omega- 6
TABLE 1. Differences in serum oxylipins between immunoglobulin A nephropathy (IgAN) patients and controls at baseline*

\begin{tabular}{|c|c|c|c|c|c|}
\hline \multirow[b]{2}{*}{ Oxylipint } & \multicolumn{2}{|c|}{ IgAN patients } & \multicolumn{2}{|c|}{ Controls } & \multirow[b]{2}{*}{$P \neq$} \\
\hline & Mean & SE & Mean & SE & \\
\hline Epoxides & 313.6 & 27.20 & 9.61 & 0.93 & $<0.001$ \\
\hline Diols & 114.0 & 6.88 & 24.74 & 2.69 & $<0.001$ \\
\hline $\operatorname{cox}$ & 238.8 & 20.14 & 22.45 & 2.09 & $<0.001$ \\
\hline 5-LOX & 47,560 & 4,372 & 144.8 & 18.81 & $<0.001$ \\
\hline 12/15-LOX & 52,210 & 5,998 & 342.6 & 45.99 & $<0.001$ \\
\hline Total oxylipins & 103,600 & 11,190 & 566.8 & 66.38 & $<0.001$ \\
\hline
\end{tabular}

TABLE 3. Differences in serum oxylipin categories between patients whose kidney function was either nonimproved or improved*

\begin{tabular}{|c|c|c|c|c|c|}
\hline \multirow[b]{2}{*}{ Oxylipint } & \multicolumn{2}{|c|}{ Nonimproved } & \multicolumn{2}{|c|}{ Improved } & \multirow[b]{2}{*}{$P \neq$} \\
\hline & Mean & SE & Mean & SE & \\
\hline 5-LOX§ & 54,790 & 6,346 & 39,330 & 5,752 & 0.06 \\
\hline $\operatorname{cox} \pi$ & 358.0 & 43.99 & 243.5 & 33.18 & 0.02 \\
\hline
\end{tabular}

* Data are means and SE for oxylipin categories significantly different between patients whose kidney function was either nonimproved $(>-25 \%$ change in UP/C ratio) or improved ( $\leq-25 \%$ change in UP/C ratio) from baseline to 2 years.

† LOX = lipoxygenase; COX = cyclooxygenase.

₹ Student's $t$-tests comparing two groups.

$\S$ Total 5-LOX values measured at baseline (presupplementation).

१ी Total COX values measured postsupplementation. 
to omega-3 fatty acids. More studies are needed to determine the normal ranges of oxylipins as well as the deviations from these normal ranges in different disease states, so that dietary interventions can be evaluated. The long-term goal is the ability to design targeted nutritional recommendations for health optimization and disease prevention in individuals and groups.

Omega-3 fatty acids are generally deficient in Western diets. This deficiency is due in part to the disproportionately high consumption of omega- 6 fatty acids, which are more common in commercial products like fast food, as compared to omega-3 fatty acids, which are less common. Dietary deficiencies in omega-3 fatty acids may be contributing to the preponderance of inflammatory diseases in the United States and elsewhere.

\section{References}

Ballantyne CM. 1999. Reducing atherothrombotic events in high-risk patients: Recent data on therapy with statins and fatty acids. Curr Atheroscler Rep 1:6-8.

Bouwens M, van de Rest O, Dellschaft N, et al. 2009. Fishoil supplementation induces antiinflammatory gene expression profiles in human blood mononuclear cells. Am J Clin Nutr 90:415-24.

Burdge GC, Calder PC. 2006. Dietary al pha-linolenic acid and health-related outcomes: A metabolic perspective. Nutr Res Rev 19:26-52.

Capanni M, Calella F, Biagini MR, et al. 2006. Prolonged n-3 polyunsaturated fatty acid supplementation ameliorates hepatic steatosis in patients with non-alcoholic fatty liver disease: A pilot study. Aliment Pharmacol The 23:1143-51.

De Caterina R, Madonna R, Massaro M. 2004. Effects of omega-3 fatty acids on cytokines and adhesion molecules. Curr Atheroscler Rep 6:485-91.

Donadio JV, Bergstralh EJ, Offord KP, et al. 1994. Clinica and histopathologic associations with impaired renal function in lgA nephropathy. Mayo Nephrology Collaborative Group. Clin Nephrol 41:65-71.

[EAS] European Atherosclerosis Society. 1987. Strategies for the prevention of coronary heart disease: A policy statement of the European Atherosclerosis Society. Eur Heart J 8:77-88.

Fassett RG, Gobe GC, Peake JM, Coombes JS. 2010 Omega-3 polyunsaturated fatty acids in the treatment of kidney disease. Am J Kidney Dis 56:728-42.

Freemantle E, Vandal M, Tremblay-Mercier J, et al. 2006 Omega-3 fatty acids, energy substrates and brain function during aging. Prostaglandins Leukot Essent Fatty Acids 75:213-20.

Funk CD. 2001. Prostaglandins and leukotrienes: Advances in eicosanoid biology. Science 294:1871-5.

Goldberg RJ, Katz J. 2007. A meta-analysis of the analgesic effects of omega-3 polyunsaturated fatty acid supplementation for inflammatory joint pain. Pain 129:210-23.

Goyens PL, Spilker ME, Zock PL, et al. 2006. Conversion of alpha-linolenic acid in humans is influenced by the $a b$ solute amounts of alpha-linolenic acid and linoleic acid

in the diet and not by their ratio. Am J Clin Nutr 84:44-53.
The effects of omega- 3 fatty acids on modulating inflammation are poorly understood, in part due to the lack of information regarding the plasma concentrations of omega- 3 fatty acids and their oxylipin products in both healthy and diseased states. However, omega-3 fatty acids have well-supported broad effects on human health; they improve cardiovascular health, improve the condition of patients suffering from autoimmune and neurological diseases, and promote renal health.

Though in general we can conclude that omega-3 fatty acids are beneficial to health, a better understanding of the mechanisms and individual variations in the metabolism of the omega- 3 fatty acids and their oxylipin products is needed. With this understanding, nutritional recommendations regarding the intakes of these anti-inflammatory dietary compounds could be improved to better treat and prevent inflammatory diseases.

A.M. Zivkovic is Associate Director of Scientific Development and Translation, Foods for Health Institute, Department of Food Science and Techno/ogy, UC Davis; N. Telis is Undergraduate Research Assistant, Foods for Health Institute, UC Davis; J.B. German is Director, Foods for Health Institute, and Professor, Department of Food Science and Technology, UC Davis; and B.D. Hammock is Distinguished Professor, Department of Entomology, UC Davis.

Robert M. Hackman, Research Nutritionist in the UC Davis Department of Nutrition, served as Guest Associate Editor for this article. This research was funded by the Center for Health and Nutrition Research (CHNR) Pilot Grant Program, UC Discovery Program (05GEB01NHB), the National Institute of Environmental Health Sciences (P42ES004699), the California Dairy Research Foundation and the CHARGE study (P01 ES11269).
Griffin MD, Sanders TAB, Davies IG, et al. 2006. Effects of altering the ratio of dietary n- 6 to $n-3$ fatty acids on insulin sensitivity, lipoprotein size and postprandial lipemia in men and postmenopausal women aged $45-70 \mathrm{y}$ : The OPTILIP Study. Am J Clin Nutr 84:1290-8.

Harris WS, Mozaffarian D, Lefevre M, et al. 2009. Towards establishing dietary reference intakes for eicosapentaenoic and docosahexaenoic acids. J Nutr 139:804-19S.

Hogg RJ, Lee J, Nardelli N, et al. 2006. Clinical trial to evaluate omega-3 fatty acids and alternate day prednisone in patients with IgA nephropathy: Report from the Southwest Pediatric Nephrology Study Group. Clin J Am Soc Nephrol 1:467-74

Hooper L, Thompson RL, Harrison RA, et al. 2004. Omega 3 fatty acids for prevention and treatment of cardiovas cular disease. Cochrane Database Syst Rev CD003177. Horrocks LA, Yeo YK. 1999. Health benefits of docosahexaenoic acid (DHA). Pharmacol Res 40:211-25. Imig JD, Zhao X, Zaharis CZ, et al. 2005. An orally active epoxide hydrolase inhibitor lowers blood pressure and provides renal protection in salt-sensitive hypertension. Hypertension 46:975-81.

Jedrychowski W, Perera F, Rauh V, et al. 2007. Fish intake during pregnancy and mercury level in cord and maternal blood at delivery: An environmental study in Poland. Int J Occup Med Environ Health 20:31-7.

Kris-Etherton PM, Taylor DS, Yu-Poth S, et al. 2000. Polyunsaturated fatty acids in the food chain in the United States. Am J Clin Nutr 71:179-88S.

Liou YA, King DJ, Zibrik D, Innis SM. 2007. Decreasing linoleic acid with constant alpha-linolenic acid in dietary fats increases ( $\mathrm{n}-3$ ) eicosapentaenoic acid in plasma phospholipids in healthy men. J Nutr 137:945-52.

McNamara RK, Jandacek R, Rider T, et al. 2007. Abnormalities in the fatty acid composition of the postmortem orbitofrontal cortex of schizophrenic patients: Gender differences and partial normalization with antipsychotic medications. Schizophrenia Res 91:37-50.

Pawlosky RJ, Hibbeln JR, Lin Y, et al. 2003. Effects of beefand fish-based diets on the kinetics of n-3 fatty acid metabolism in human subjects. Am J Clin Nutr 77:565-72.

Sanchez-Villegas A, Henríquez P, Figueiras A, et al. 2007. Long chain omega-3 fatty acids intake, fish consumption and mental disorders in the SUN cohort study. Eur J Nutr 46:337-46.
Schaefer EJ, Bongard V, Beiser AS, et al. 2006. Plasma phosphatidylcholine docosahexaenoic acid content and risk of dementia and Alzheimer disease: The Framingham Heart Study. Arch Neurol 63:1545-50.

Serhan CN, Savill J. 2005. Resolution of inflammation: The beginning programs the end. Nat Immunol 6:1191-7.

Shearer GC, Harris WS, Pedersen TL, Newman JW. 2009. Detection of omega-3 oxylipins in human plasma and response to treatment with omega-3 acid ethyl esters. J Lipid Res 51(8):2074-81.

Simopoulos AP. 2002. Omega-3 fatty acids in inflammation and autoimmune diseases. J Am Coll Nutr 21:495-505

Simopoulos AP. 2008. The importance of the omega-6/ omega-3 fatty acid ratio in cardiovascular disease and other chronic diseases. Exp Biol Med (Maywood) 233:674-88

Simopoulos AP, Leaf A, Salem N Jr. 1999. Workshop on the essentiality of and recommended dietary intakes for omega- 6 and omega-3 fatty acids. J Am Coll Nutr 18:487-9.

Stahl LL, Snyder BD, Olsen AR, Pitt JL. 2009. Contaminants in fish tissue from US lakes and reservoirs: A national probabilistic study. Environ Monit Assess 150:3-19.

Sunderland EM. 2007. Mercury exposure from domestic and imported estuarine and marine fish in the U.S. seafood market. Environ Health Perspect 115:235-42.

Thies F, Garry JM, Yaqoob P, et al. 2003. Association of $\mathrm{n}-3$ polyunsaturated fatty acids with stability of atherosclerotic plaques: A randomised controlled trial. Lancet 361:477-85.

[USDA] US Department of Agriculture. 2011. USDA Nutrient Database. www.nal.usda.gov/fnic/foodcomp/search.

Ye D, Zhang D, Oltman C, et al. 2002. Cytochrome p-450 epoxygenase metabolites of docosahexaenoate potently dilate coronary arterioles by activating largeconductance calcium-activated potassium channels. J Pharmacol Exp Ther 303:768-76.

Zainal Z, Longman AJ, Hurst S, et al. 2009. Relative efficacies of omega-3 polyunsaturated fatty acids in reducing expression of key proteins in a model system for studying osteoarthritis. Osteoarthritis Cartilage 17:896-905.

Zhao X, Yamamoto T, Newman JW, et al. 2004. Soluble epoxide hydrolase inhibition protects the kidney from hypertension-induced damage. J Am Soc Nephrol 15:1244-53. 